\title{
Using Fourier Transform Infrared Spectroscopy to Study Effects of Magnetic Field Treatment on Wheat (Triticum aestivum L.) Seedlings
}

\author{
Zhenlin Wei, Dejie Jiao, and Junxiao Xu \\ Biological Science Department, Dezhou University, Dezhou, Shandong 253023, China \\ Correspondence should be addressed to Zhenlin Wei; wzl19741028@163.com
}

Received 3 February 2015; Revised 17 March 2015; Accepted 17 March 2015

Academic Editor: Feride Severcan

Copyright (C) 2015 Zhenlin Wei et al. This is an open access article distributed under the Creative Commons Attribution License, which permits unrestricted use, distribution, and reproduction in any medium, provided the original work is properly cited.

\begin{abstract}
Magnetic field treatments have been utilized to promote germination and growth of a variety of species of plants; however the mechanism of concern has not been fully elucidated. In this research, wheat seedlings were treated with $500 \mathrm{mT}$ and $1500 \mathrm{mT}$ static magnetic field (SMF) for 10 and $20 \mathrm{~min}$, respectively. Analyzing Fourier transform infrared spectra collected from leaves of seedlings showed that SMF treatments decreased the contents of lipids and proteins, shifted bands to higher wavenumbers in $3000-$ $2800 \mathrm{~cm}^{-1}$ regions, and increased the ratio of $\mathrm{CH}_{2} / \mathrm{CH}_{3}$ which likely indicates a structural variation of lipids. For bands assigned to different second structures of proteins, slight bands shifting and changing the ratio of different second structures of proteins were observed due to SMF treatments. To summarize, the results revealed that lipids rather than proteins were sensitive to SMF treatments. The results provided insight into the SMF induced conformational changes of lipids and proteins in wheat leaves, which will help elucidate the biological mechanisms of SMF on plant growth and development.
\end{abstract}

\section{Introduction}

For decades, the effects of static magnetic fields (SMF) on plant growth have been the subject of different research studies. Magnetic field treatments influence the physiological and biochemical process in the seeds, thereby contributing to greater vigor and increased crop products. Researchers noted that aged onion (Allium cepa) and rice (Oryza sativa) seeds exposed to a weak electromagnetic field for $12 \mathrm{~h}$ increased the germination and root length of seedlings [1]. Moreover, enhancing germination and growth of Cork oak (Quercus suber) seedlings after being exposed to chronic magnetic field was reported [2]. Exposure of magnetic field (10 mT for $40 \mathrm{~h}$ ) increased plant height, seed weight, and yield of chickpea and wheat $[3,4]$. In addition to seed germination and growth, power frequency magnetic fields $(100 \mathrm{mT})$ treatment on Vicia faba seedlings enhanced mitotic index and ${ }^{3} \mathrm{H}-$ thymidine uptake [5]. Furthermore, treatment of sunflower seeds improved seed coat membrane integrity and reduced the cellular leakage and electrical conductivity, as well as achieving higher enzyme activity [6]. Increasing number of evidences indicates that SMF treatment alters apoptosis and changes gene expression [7].

The vibration of chemical bonds can absorb radiation in the middle infrared (MIR) region between 4000 and $400 \mathrm{~cm}^{-1}$, and each functional group in a molecule has characteristic absorption frequencies in the IR spectrum. Particularly, Fourier transform infrared spectra (FTIR) can be used to probe the structure of biological composition or its chemical group, and the composition and structure of molecular functional group can be determined by analyzing the position, width, and intensity of acquired spectra in a complex biological system when relying on certain algorithms [8]. The advantages of FTIR method enable it to examine the initial response to stimuli with high sensitivity through acquiring rapidly spectra from very small amounts of samples [8] and offer a fast method to fingerprint the global cellular features under specific conditions [9].

Wheat (Triticum aestivum L.) is one of the world's most important food crops; therefore SMF has been used to promote seed germination and seedling emergence [4]. Magnetic field treatment of seeds leads to acceleration of plants growth 
TABLE 1: Different treatments used in this research.

\begin{tabular}{lcc}
\hline Treatment number & Strength of magnetic field & Treatment time \\
\hline Treatment 1 & $500 \mathrm{mT}$ & $10 \mathrm{~min}$ \\
Treatment 2 & $1500 \mathrm{mT}$ & $10 \mathrm{~min}$ \\
Treatment 3 & $500 \mathrm{mT}$ & $20 \mathrm{~min}$ \\
Treatment 4 & $1500 \mathrm{mT}$ & $20 \mathrm{~min}$ \\
Control & - & $20 \mathrm{~min}$ \\
\hline
\end{tabular}

and root development and increases the activities of some enzymes such as amylase and nitrate reductase $[4,10]$, to gain high grain yield of wheat by shortening the days from sowing to complete ground cover. However, physiological mechanisms of magnetic field on plant growth are not completely understood [8]. In the current study, the effects of SMF treatments on wheat seedlings were investigated by FTIR spectroscopy to monitor the conformational changing of functional groups of proteins and lipids, thereby providing novel information on the SMF induced effects on wheat growth.

\section{Materials and Methods}

2.1. Materials. Seeds of wheat (Lumail7) were surface sterilized and incubated at $25^{\circ} \mathrm{C}$ in the dark for germination and then grew with day/night regime of $16 \mathrm{~h} / 8 \mathrm{~h}$ at $25^{\circ} \mathrm{C}$ supplied with Hoagland solutions. After 1 week of acclimation, when the seedlings reached the stage of completely developed first trifoliolate, they were transferred to the fresh nutrient solution and subject to SMF treatments. The SMF treatments were conducted according to Liu and Yu [11]. Briefly, a TYU$2000 \mathrm{H}$ double yoke water-cooled electromagnet (Tianyu Company, China) was utilized to treat the seedlings cultured in nutrient solution with conditions listed in Table 1, and each treatment was duplicated three times. Hence, the seedlings were grown in same nutrient solution for 14 days before FTIR analysis.

2.2. FTIR Spectroscopy Analyzing. FTIR assay was conducted according to our previous paper [12]. All spectra were normalized and baseline-corrected with Nicolet Omnic 7 software and smoothed with a smoothing factor of 9. The spectra for control and SMF treated samples were calculated from 3 repeats and each used a different individual. These replicates were averaged and these averaged spectra for each sample were then used for statistical analysis.

2.3. Data Analysis. Since the original spectra yield rather broad bands in specific regions, the second-derivative analysis has to be applied to resolve the fine details of interesting bands with bandwidth of 20.0 and enhancement of 3.0 [13, 14]. Second-derivative spectra were generated using 11-point Savitzky-Golay algorithm. Curve-fitting of the spectra was performed with PeakFit V4.12 software with default parameters; thereby the precise position and the area of band were obtained as the results. Statistical analysis was performed using SPSS 11.5 software. Differences were analyzed by paired

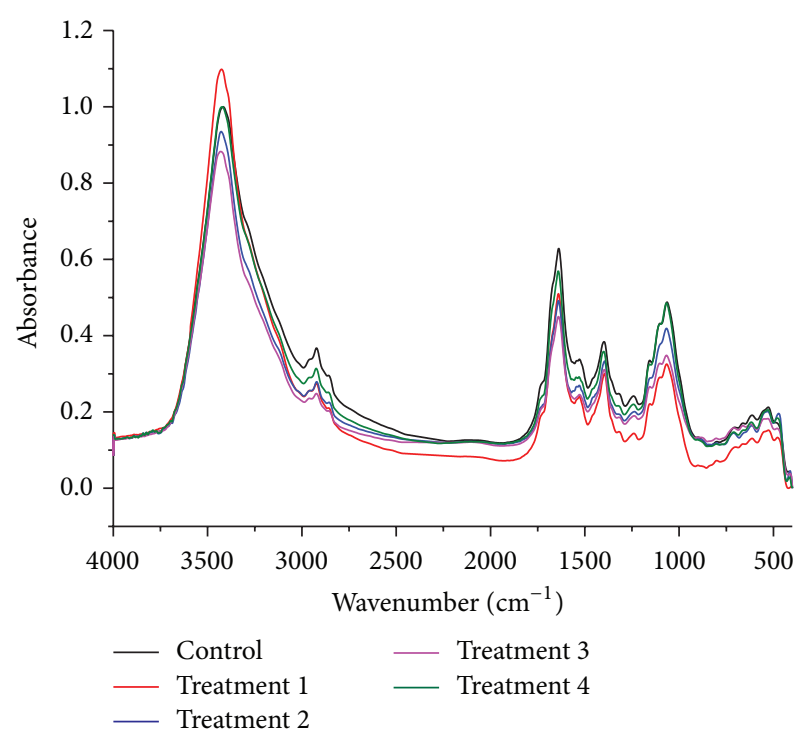

FIGURE 1: Spectra of control and treated wheat leaves in the wavenumber of $4000-400 \mathrm{~cm}^{-1}$.

$t$-test. A probability level ( $P$ value) of less than 0.05 is regarded as significant.

\section{Results and Discussion}

3.1. Infrared Spectra from Control and Magnetic Field Treated Samples. In this research, FTIR spectra were acquired to detect the conformational changes and content variations of the functional groups contributed from protein and lipids of leaves. The average spectra in the $4000-400 \mathrm{~cm}^{-1}$ wavenumber region of control and treated samples were shown in Figure 1.

Generally, the spectra consist of several bands arising from the vibration of different groups belonging to proteins, lipids, carbohydrates, and nucleic acids, indicating that the leaves are rich in biochemical compositions [15]. Specifically, the band located around $3295 \mathrm{~cm}^{-1}$ represents $\mathrm{N}-$ $\mathrm{H}$ stretching vibrations that are mainly caused by proteins $[13,16]$. The bands between 3000 and $2800 \mathrm{~cm}^{-1}$ mainly represent $\mathrm{C}-\mathrm{H}$ stretching vibrations that are caused by lipids [15]. Protein absorption bands including amide I and amide II bands that mainly located between 1700 and $1500 \mathrm{~cm}^{-1}$ were overlapped with other absorption bands within this region and appeared several prominent bands. The bands between 1500 and $1000 \mathrm{~cm}^{-1}$ were of "fingerprint" region [17]; amide III and the functional groups of nucleic acid and carbohydrates contributed to these absorption bands in samples. Overall, the spectrum of control and magnetic field treated samples differ in the figure of the absorption spectrum, indicating obvious changes in the structure and contents of biological components due to SMF.

3.2. Comparative Analysis of Second-Derivative Spectra from Control and SMF Treated Samples. The original spectra faced complex multicomponent bands which overlapped into 
TABLE 2: Curve-fitting results of bands in $3000-2800 \mathrm{~cm}^{-1}$ region.

\begin{tabular}{lcccccccccccccc}
\hline Group & $\mathrm{C}$ & $\mathrm{SD}$ & Treatment 1 & $\mathrm{SD}$ & $P$ & Treatment 2 & $\mathrm{SD}$ & $P$ & Treatment 3 & SD & $P$ & Treatment 4 & SD & $P$ \\
\hline sym $\mathrm{CH}_{2}$ & 15.9 & 0.36 & 15.26 & 0.36 & 0.01 & 18.69 & 0.18 & 0.01 & 15.69 & 0.36 & 0 & 12.50 & 0.36 & 0 \\
sym $\mathrm{CH}_{3}$ & 19.62 & 1.44 & 18.29 & 0.18 & 0.04 & 12.95 & 0.18 & 0.02 & 18.56 & 0.36 & 0.04 & 22.09 & 1.98 & 0 \\
asym $\mathrm{CH}_{2}$ & 43.7 & 5.58 & 46.49 & 3.78 & 0.04 & 45.17 & 4.5 & 0.14 & 49.46 & 6.3 & 0.07 & 46.54 & 3.24 & 0.04 \\
asym $\mathrm{CH}_{3}$ & 20.78 & 1.08 & 19.96 & 0.54 & 0.01 & 23.18 & 0.54 & 0.01 & 16.29 & 0.18 & 0 & 18.87 & 0.54 & 0.01 \\
\hline
\end{tabular}

Note: sym $\mathrm{CH}_{2}: 2860-2840 \mathrm{~cm}^{-1}$; sym CH $3: 2885-2860 \mathrm{~cm}^{-1}$; asym $\mathrm{CH}_{2}: 2945-2905 \mathrm{~cm}^{-1}$; asym CH $\mathrm{CH}_{3}: 2990-2945 \mathrm{~cm}^{-1}$; C: control.

a broad unresolved absorption, and the individual component absorption from condensed phase spectra cannot be resolved by increasing spectral resolution, causing difficulty in bands differentiation and their assignment. Therefore, the widely used second-derivative spectra and curve-fitting processes were performed to distinguish overlapped peaks and achieve more precise calculation on bands position and their area. The advantage of this process lies in that the data is denoised to a great extent and overlapping is largely minimized [15].

As shown in Figure 1, compared with the control, there is an overall decrease in the intensity of absorption bands of treatments 2, 3, and 4 and an increase in intensity of absorption bands for treatment 1 . These changes in absorption of specific vibrational bands indicated changes in concentrations of proteins, lipids, and phospholipids of the samples due to SMF treatment.

Analysis of the second-derivative spectra of control and SMF treated samples revealed several differences, including peak location shifting and peak height variation, especially in the regions from 1500 to $3500 \mathrm{~cm}^{-1}$, demonstrating that obvious conformational changes occurred for lipids and proteins. The spectrum of treatment 1 was more similar to control than others, demonstrating that the higher strength of SMF treatment induced more obvious variations on spectra.

\subsubsection{Changes in Peak around $3014 \mathrm{~cm}^{-1}$. The region 3300 to} $3000 \mathrm{~cm}^{-1}$ is characteristic for $\mathrm{C}-\mathrm{H}$ stretching vibrations of $\mathrm{C} \equiv \mathrm{C}, \mathrm{C}=\mathrm{C}$, and $\mathrm{Ar}-\mathrm{H}$, while the region from 3000 to 2700 is dominated by the $\mathrm{C}-\mathrm{H}$ stretching vibrations of $-\mathrm{CH}_{3}, \mathrm{CH}_{2}$, $\mathrm{CH}$, and $\mathrm{CHO}$ functional groups, respectively $[17,18]$. In this research, the bands within this region were quantitatively analyzed to uncover the changes of lipids (Table 2).

A weak intense band was found around $3014 \mathrm{~cm}^{-1}$ in all spectra, which was assigned to the vibrating stretching of cisalkene $(\mathrm{HC}=\mathrm{CH})$ group which belongs to polyunsaturated lipids $[19,20]$, and the existence of this peak indicated that leaf is rich in several types of polyunsaturated lipids. Yoshida et al. suggested that the appearance of $3014 \mathrm{~cm}^{-1}$ peak indicates the enrichment of docosahexaenoic acid (DHA) [20]. The comparative spectra of control with those of different treatments showed similar bandwidth and obviously peak positions shifting, suggesting the changes of polyunsaturated fatty acid species. Furthermore, it seems that more intensive treatment induced more obviously peak shifting, ranging from $3014.3 \mathrm{~cm}^{-1}$ (control) to $3020.8 \mathrm{~cm}^{-1}$ (treatment 4). This result implied that the composition of leave unsaturated lipids was SMF treatment sensitivity.
The area of this band of control was much higher than that of treatments based on curve-fitting analyzing, that was 0.1602 for control and $0.051(P<0.05)$ for treatment 3 . As this band is a useful indicator of the different degrees of unsaturation in acyl chains of phospholipids, the results implied that SMF treatments reduced contents and varied components of unsaturated lipids.

3.2.2. Changes in $3020-2800 \mathrm{~cm}^{-1}$ Region. It has been evidenced that the band of this region mainly represents the $\mathrm{CH}_{3}$ and $\mathrm{CH}_{2}$ groups of lipids, since absorption in 2990$2945 \mathrm{~cm}^{-1}$ region is derived from asymmetric stretch of $\mathrm{CH}_{3}$ group, in $2945-2905 \mathrm{~cm}^{-1}$ from asymmetric stretch of $\mathrm{CH}_{2}$, in $2885-2860 \mathrm{~cm}^{-1}$ from symmetric stretch of $\mathrm{CH}_{3}$, and in $2860-2840 \mathrm{~cm}^{-1}$ from symmetric stretch of $\mathrm{CH}_{2}$ [21]. Within this region, four prominent bands were revealed in control which located at 2957.8, 2920.6, 2883.3, and $2851.8 \mathrm{~cm}^{-1}$. The dominant bands around 2957.8 and $2883.3 \mathrm{~cm}^{-1}$ were assigned to $\mathrm{CH}_{3}$ stretching vibration, while bands around 2920.6 and $2851.8 \mathrm{~cm}^{-1}$ were assigned to $\mathrm{CH}_{2}$ stretching vibration [17].

With correlation with effect on bands around $3014 \mathrm{~cm}^{-1}$, SMF treatments on other bands in 3000 to $2800 \mathrm{~cm}^{-1}$ region were also different to that of control. After SMF treatments, four main bands were distinguished in treatment 1 , six bands in treatments 2 and 4 , and five bands in treatment 3 (Table 2). Regarding the peak shifting, the bands were shifted to 2851.6, 2921.2 , and $2959.1 \mathrm{~cm}^{-1}$, respectively. With increasing of the strength of treatment, the composing of bands was changed more obviously. First, band at $2957.8 \mathrm{~cm}^{-1}$ was shifted to higher wavenumber in treatments 2,3 , and 4 , and the highest localization was observed in treatment 4 at $3969.6 \mathrm{~cm}^{-1}$, while bands at 2851.8 and 2883.3 almost remained stable in all treatments. This result indicated obvious structural variations of this band subject to magnetic field treatment.

Second, compared with control, two, one, and two newly emerged bands were uncovered in spectra from treatments 2 to 4 , respectively (Table 2). The small band at $2903.1 \mathrm{~cm}^{-1}$ in treatment 2 is due to the Fermi resonance of the $\mathrm{CH}_{2}$ symmetric stretch, and the weak band centered around $2953 \mathrm{~cm}^{-1}$ represents $\mathrm{CH}_{3}$ terminal methyl stretch [22]. The peak centered at $2942 \mathrm{~cm}^{-1}$ in spectra of treatment 4 is a combination of two resonances: the $\mathrm{CH}_{3}$ Fermi resonance and the $\mathrm{CH}_{3}$ asymmetric stretch at $2969.6 \mathrm{~cm}^{-1}$ [23]. All above results demonstrated that SMF treatments resulted in structural variation of lipids and this effect likely contributed to its physiological roles [24]. 
TABLE 3: Curve-fitting results of bands in $1700-1800 \mathrm{~cm}^{-1}$ region.

\begin{tabular}{lcccccccccccccc}
\hline Group & C & SD & Treatment 1 & SD & $P$ & Treatment 2 & SD & $P$ & Treatment 3 & SD & $P$ & Treatment 4 & SD & $P$ \\
\hline$\beta$-sheets & 47.38 & 1.75 & 47.95 & 4.20 & 0.245 & 49.3 & 2.73 & 0.01 & 40.08 & 1.19 & 0.01 & 47.44 & 10.360 & 0.72 \\
$\alpha$-helices & 35.26 & 3.50 & 36.96 & 2.10 & 0.047 & 35.23 & 3.92 & 0.37 & 44.12 & 1.95 & 0.19 & 35.76 & 3.78 & 0.01 \\
Turn & 13.6 & 0.84 & 11.81 & 1.47 & 0.01 & 12.14 & 0.56 & 0.02 & 12.40 & 0.80 & 0.02 & 13.23 & 0.42 & 0.08 \\
Inter $\beta$-sheets & 3.76 & 0.21 & 3.28 & 0.14 & 0.043 & 3.34 & 0.42 & 0.08 & 3.391 & 0.343 & 0 & 3.57 & 0.07 & 0.13 \\
\hline
\end{tabular}

Overall, the area of bands located in $2800-3000 \mathrm{~cm}^{-1}$ of all treatments was less than control of which treatment 3 was the smallest one $(P<0.05)$ (Table 2$)$. Notably, SMF treatment increased the percent of bands assigned to the $\mathrm{CH}_{2}$ group. The area of bands at 2851 and $2920 \mathrm{~cm}^{-1}$ accounted for $59.60 \%$ of total area of bands in $2800-3000 \mathrm{~cm}^{-1}$ in control, and this value reached $61.75 \%$ in treatment $1(P<$ $0.05), 63.87 \%$ in treatment $2,65.14 \%$ in treatment 3 , and $59.04 \%$ in treatment 4 [25]. Therefore, the curve-fitting results revealed that magnetic field treatments utilized in this research increased the $\mathrm{CH}_{2} / \mathrm{CH}_{3}$ ratio with exception for treatment 4 , an indicator which reflected the increased order status of lipids. The increased $\mathrm{CH}_{2} / \mathrm{CH}_{3}$ ratio is also a sign of higher fatty acyl chain unsaturation, which has been known to occur with SMF treatment. These results were in well agreement with conclusions that the magnetic field induced lipid unsaturation and slowed down the process of the peroxidation of lipids [26-28].

The observations suggested that SMF induced changes in the conformational order of lipid acyl chains. It is well known that the $\mathrm{CH}_{2}$ antisymmetric and symmetric stretching vibrations give information about the state-of-order of the hydrocarbon tails in lipids [29]. The appearance of increasing in the frequencies of these bands with SMF treatment indicates the functions of disordering the lipid system by increasing the number of $\mathrm{CH}_{3}$ groups [9]. On the other hand, the increased ratio of $\mathrm{CH}_{2} / \mathrm{CH}_{3}$ reflected the transformation of conformation of lipid tails [30].

Our results showed that SMF treatments increased ratio of $\mathrm{CH}_{2} / \mathrm{CH}_{3}$ and significantly varied constitutions of components of lipids. It was suggested that SMF induced advantageous and adverse effects upon lipid metabolism, and the higher strength of treatment resulted in more obvious appearance. Since these lipid components could be divided into plasma membrane related and energy metabolism related, SMF induced effect on lipids would involve a wide range of biological processes, which need to be experimentally investigated further.

3.3. Changes in Amide I Region. The amide I band of proteins is located in the region from 1700 to $1600 \mathrm{~cm}^{-1}$, which is primarily due to the $\mathrm{C}=\mathrm{O}$ stretching vibration of the amide groups in weakly coupling with the in-plane $\mathrm{N}-\mathrm{H}$ bending and $\mathrm{C}-\mathrm{N}$ stretching. The amide $\mathrm{I}$ band of proteins is a complex composite which consists of a number of component bands in terms of $\alpha$-helices, $\beta$-sheets (parallel pleated sheet and antiparallel pleated sheet), turns, and random-coil structures [15]. The frequency of the absorption maximum of these bands is conformationally sensitive; therefore SMF treatment induced shifts of peaks provided information about structural changes in the proteins of samples. According to methods described by Palaniappan and Dumas [17, 31], the whole amide I area was analyzed by means of curve-fitting.

Six bands were found between 1700 and $1600 \mathrm{~cm}^{-1}$ in spectra of control and treatments 1,2 , and 4 and 5 bands of treatment 3 (Table 3), and the band positions were similar to each other among all spectra, indicating that protein structure was insensitive to SMF treatments. Bands around $1610 \mathrm{~cm}^{-1}, 1620$, and $1635 \mathrm{~cm}^{-1}$ were assigned to $\beta$ sheet, bands around 1687 and $1617 \mathrm{~cm}^{-1}$ to intermolecular antiparallel $\beta$ sheet [32], and bands at $1656 \mathrm{~cm}^{-1}$ to $\alpha$-helix, while bands at $1676 \mathrm{~cm}^{-1}$ were assigned to turn structure.

The previous study reported that low magnetic field increased the area of band around $1610 \mathrm{~cm}^{-1}$, and more strength in SMF resulted in peak shifting to higher wavenumber which indicates a conformational changing of proteins [33]. Similar to their observations, the proportion of area of $1610 \mathrm{~cm}^{-1}$ band was increased in all treatments compared with control, ranging from $1.73 \%$ in control to $1.88 \%$ in treatment $2(P<0.05)$. This indicated that the area of this band in control was smaller than that of treatments 2 and 1 $(P<0.05)$. Furthermore, the area of band around $1676 \mathrm{~cm}^{-1}$ was decreased in all treatments (Table 3 ), from $13.60 \%$ in control to $12.40 \%$ in treatment $3(P<0.05)$. In addition, the ratio of $\alpha$-helices/ $\beta$-sheet was increased following SMF treatment, giving the value from $68.95 \%$ in control to $72.15 \%$ in treatment $1(P<0.05), 101.50 \%(P<0.05)$ in treatment 3 , and $70.10 \%$ in treatment $4(P<0.05)$. Based on these results, it was supposed that SMF resulted in diversity of protein species which is in well agreement with conclusion of transcriptomic analysis [7].

However, we could not observe significant peak shifting in this region which has been indicated by She et al. [34]. In proteins, the most important hydrogen bonds are those between peptide bonds. Generally, the positions of amide I bands reflect the degree of hydrogen bonding: the higher the wavenumber, the weaker the $\mathrm{H}$ bonding (i.e., the less-ordered protein structure). Therefore, the position shifting of amide I bands to higher wavenumber is a useful indicator of the overall protein disordering under experimental conditions [14]. Therefore, our results regarding amide I curve-fitting revealed that the conformation of proteins was insensitive to SMF treatments. Alternatively, this could be due to the fact that different species were diversity in the context of sensitivity to SMF; for example, the bands in amide I region in Escherichia coli were vigorously varied responding to 
10.0 T SMF, while slight changes were found in Staphylococcus aureus [34]. The effects of SMF with different intensities should be investigated further.

Based on the curve-fitting results, it was suggested that, under experimental conditions, the leaf proteins were less sensitive than lipids to SMF, since the conformational diversity of proteins was more constant than that of lipids. It should be noted that the study on conformational studies on proteins was conducted immediately treated by SMF [33], while this research was carried out two weeks later after SMF treatments. Therefore, it was likely that SMF indeed induced obvious effects on second structural transformation of proteins and these were attenuated in the interval between SMF treatments and FTIR analysis. Therefore, effects of SMF on protein structure need more analysis.

\section{Conclusion}

In conclusion, analysis of the FTIR spectra collected from leaves revealed that lipids rather than proteins were sensitive to SMF treatments. The results of the current study provided insight on the SMF induced conformational changes of lipids and proteins, as well as on the content variation of these components. The results showed that SMF treatment decreased the contents of lipids and proteins in the leaves, resulting in band shifting to higher wavenumbers in 3000 $2800 \mathrm{~cm}^{-1}$ region, as well as increasing the ratio of $\mathrm{CH}_{2} / \mathrm{CH}_{3}$ and alerting the species of unsaturated lipids. The results also implied that structural changing of lipids and proteins would be beneficial to effects of SMF treatment.

From a physiological aspect, the FTIR analysis constructs a direct link between the functional biomolecular and the physiological status under SMF treatments, because the macromolecular characteristics and their contents are fundamental factors related to several physiological processes whereby the cell promotes or declines its normal development and growth. FTIR spectroscopy offers a fast and efficient tool for detection of qualitative and quantitative SMF induced changes in the context of molecular structure constitutions in wheat leaves. In addition, it has the potential as an accurate and sensitive technique for research on physiological effects of SMF by means of detecting the conformational changing of biological molecular components.

\section{Conflict of Interests}

The authors declare that there is no conflict of interests regarding the publication of this paper.

\section{Acknowledgments}

This research is supported by the Foundation of Assets and Instruments Management of Dezhou University. The authors thank Mr. Liu Guizhong and Dr. Xu Chenshan for their technological assistances.

\section{References}

[1] M. P. Alexander and S. D. Doijode, Electromagnetic Field, A Novel Tool to Increase Germination and Seedling Vigour of Conserved Onion (Allium cepa L.) and Rice (Oryza sativa L.) Seeds with Low Viability, vol. 104 of FAO/IBPGR PGR NL, 1995.

[2] C. Celestino, M. L. Picazo, M. Toribio, J. A. Alvarez-Ude, and J. L. Bardasano, "Influence of $50 \mathrm{~Hz}$ electromagnetic fields on recurrent embryogenesis and germination of cork oak somatic embryos," Plant Cell, Tissue and Organ Culture, vol. 54, no. 1, pp. 65-69, 1998.

[3] N. A. R. Tahir and H. F. H. Karim, "Impact of magnetic application on the parameters related growth of chickpea (Cicer arietinum L.)," Jordan Journal of Biological Sciences, vol. 3, no. 4, pp. 175-184, 2010.

[4] A. Gholami and S. Sharafi, "Effect of magnetic field on seed germination of two wheat cultivars," World Academy of Science, Engineering and Technology, vol. 62, pp. 279-282, 2010.

[5] P. Rajendra, H. Sujatha Nayak, R. B. Sashidhar et al., "Effects of power frequency electromagnetic fields on growth of germinating Vicia faba L., the broad bean," Electromagnetic Biology and Medicine, vol. 24, no. 1, pp. 39-54, 2005.

[6] A. Vashisth and S. Nagarajan, "Effect on germination and early growth characteristics in sunflower (Helianthus annuus) seeds exposed to static magnetic field," Journal of Plant Physiology, vol. 167, no. 2, pp. 149-156, 2010.

[7] D. M. Binninger and V. Ungvichian, "Effects of $60 \mathrm{~Hz} \mathrm{AC}$ magnetic fields on gene expression following exposure over multiple cell generations using Saccharomyces cerevisiae," Bioelectrochemistry and Bioenergetics, vol. 43, no. 1, pp. 83-89, 1997.

[8] L. Corte, P. Rellini, L. Roscini, F. Fatichenti, and G. Cardinali, "Development of a novel, FTIR (Fourier transform infrared spectroscopy) based, yeast bioassay for toxicity testing and stress response study," Analytica Chimica Acta, vol. 659, no. 12, pp. 258-265, 2010.

[9] A. Alvarez-Ordóñez, J. Halisch, and M. Prieto, "Changes in Fourier transform infrared spectra of Salmonella enterica serovars Typhimurium and Enteritidis after adaptation to stressful growth conditions," International Journal of Food Microbiology, vol. 142, no. 1-2, pp. 97-105, 2010.

[10] L. Kordas, "The effect of magnetic field on growth, development and the yield of spring wheat," Polish Journal of Environmental Studies, vol. 11, no. 5, pp. 527-530, 2002.

[11] Q. X. Liu and L. C. Yu, "Effect of the treatment of magnetic field on the seed vigor and the enzymatic activity of POD and SOD in leaf of Cabbage," Journal of Anhui Agricultural Sciences, vol. 25, pp. 13626-13627, 2010.

[12] Z. L. Wei, L. Dong, and Z. H. Tian, "Fourier transform infrared spectrometry study on early stage of cadmium stress in clover leaves," Pakistan Journal of Botany, vol. 41, no. 4, pp. 1743-1750, 2009.

[13] W. F. Wolkers, A. Bochicchio, G. Selvaggi, and F. A. Hoekstra, "Fourier transform infrared microspectroscopy detects changes in protein secondary structure associated with desiccation tolerance in developing maize embryos," Plant Physiology, vol. 116, no. 3, pp. 1169-1177, 1998.

[14] J. Yang and H. E. Yen, "Early salt stress effects on the changes in chemical composition in leaves of ice plant and Arabidopsis. A fourier transform infrared spectroscopy study," Plant Physiology, vol. 130, no. 2, pp. 1032-1042, 2002.

[15] L. D’Souza, P. Devi, M. P. Divya Shridhar, and C. G. Naik, "Use of Fourier Transform Infrared (FTIR) spectroscopy to study 
cadmium-induced changes in Padina tetrastromatica (Hauck)," Analytical Chemistry Insights, vol. 2008, no. 3, pp. 135-143, 2008.

[16] P. L. R. M. Palaniappan and V. Vijayasundaram, "The FTIR study of the brain tissue of Labeo rohita due to arsenic intoxication," Microchemical Journal, vol. 91, no. 1, pp. 118-124, 2009.

[17] P. R. Palaniappan and V. B. Renju, "FT-IR study of the effect of zinc exposure on the biochemical contents of the muscle of Labeo rohita," Infrared Physics and Technology, vol. 52, no. 1, pp. 37-41, 2009.

[18] N. G. Howlett and S. V. Avery, "Induction of lipid peroxidation during heavy metal stress in Saccharomyces cerevisiae and influence of plasma membrane fatty acid unsaturation," Applied and Environmental Microbiology, vol. 63, no. 8, pp. 2971-2976, 1997.

[19] M. D. Guillén and N. Cabo, "Usefulness of the frequency data of the Fourier transform infrared spectra to evaluate the degree of oxidation of edible oils," Journal of Agricultural and Food Chemistry, vol. 47, no. 2, pp. 709-719, 1999.

[20] S. Yoshida, Q.-Z. Zhang, S. Sakuyama, and S. Matsushima, "Metabolism of fatty acids and lipid hydroperoxides in human body monitoring with Fourier transform Infrared Spectroscopy," Lipids in Health and Disease, vol. 8, article 28, 2009.

[21] S. Sirikwanpong, W. Dahlan, S. Ngamukote et al., "The alterations of erythrocyte phospholipids in type 2 diabetes observed after oral high-fat meal loading: the FTIR spectroscopic and mass spectrometric studies," Journal of Clinical Biochemistry and Nutrition, vol. 47, no. 2, pp. 111-120, 2010.

[22] D. Borchman, G. N. Foulks, M. C. Yappert, D. Tang, and D. V. Ho, "Spectroscopic evaluation of human tear lipids," Chemistry and Physics of Lipids, vol. 147, no. 2, pp. 87-102, 2007.

[23] J. Liu and J. C. Conboy, "Structure of a gel phase lipid bilayer prepared by the Langmuir-Blodgett/Langmuir-Schaefer method characterized by sum-frequency vibrational spectroscopy," Langmuir, vol. 21, no. 20, pp. 9091-9097, 2005.

[24] S. Y. Lin, K. J. Duan, and T. C. Lin, "Direct or indirect skin lipidordering effect of pyrrolidone carboxylate sodium after topical treatment with penetration enhancers," Biomedical Materials and Engineering, vol. 5, no. 1, pp. 9-20, 1995.

[25] E. G. Trams, W. L. Stahl, and J. Robinson, "Formation of Sacyl pantetheine from acyl-coenzyme A by plasma membranes," BBA - Biomembranes, vol. 163, no. 4, pp. 472-482, 1968.

[26] E. Ciejka and A. Goraç, "Influence of low magnetic field on lipid peroxidation," Polski Merkuriusz Lekarski, vol. 24, no. 140, pp. 106-108, 2008.

[27] G. V. Novitskaya, O. A. Tserenova, T. K. Kocheshkova, and Y. I. Novitskii, "Effect of alternating magnetic field on the composition and level of lipids in radish seedlings," Russian Journal of Plant Physiology, vol. 53, no. 1, pp. 75-84, 2006.

[28] M. Mihoub, A. El May, A. Aloui, A. Chatti, and A. Landoulsi, "Effects of static magnetic fields on growth and membrane lipid composition of Salmonella typhimurium wild-type and dam mutant strains," International Journal of Food Microbiology, vol. 157, no. 2, pp. 259-266, 2012.

[29] S. B. Akkas, M. Severcan, O. Yilmaz, and F. Severcan, "Effects of lipoic acid supplementation on rat brain tissue: an FTIR spectroscopic and neural network study," Food Chemistry, vol. 105, no. 3, pp. 1281-1288, 2007.

[30] I. Prudovsky, T. K. Suresh Kumar, S. Sterling, and D. Neivandt, "Protein-phospholipid interactions in nonclassical protein secretion: Problem and methods of study," International Journal of Molecular Sciences, vol. 14, no. 2, pp. 3734-3772, 2013.
[31] P. Dumas and L. Miller, "The use of synchrotron infrared microspectroscopy in biological and biomedical investigations," Vibrational Spectroscopy, vol. 32, no. 1, pp. 3-21, 2003.

[32] E. Saguer, N. Fort, P. A. Alvarez, J. Sedman, and A. A. Ismail, "Structure-functionality relationships of porcine plasma proteins probed by FTIR spectroscopy and texture analysis," Food Hydrocolloids, vol. 22, no. 3, pp. 459-467, 2008.

[33] T. Ikehara, H. Yamaguchi, K. Hosokawa, H. Miyamoto, and K. Aizawa, "Effects of ELF magnetic field on membrane protein structure of living Hela cells studied by fourier transform infrared spectroscopy," Bioelectromagnetics, vol. 24, no. 7, pp. 457-464, 2003.

[34] Z. She, X. Hu, X. Zhao, Z. Ren, and G. Ding, "FTIR investigation of the effects of ultra-strong static magnetic field on the secondary structures of protein in bacteria," Infrared Physics and Technology, vol. 52, no. 4, pp. 138-142, 2009. 

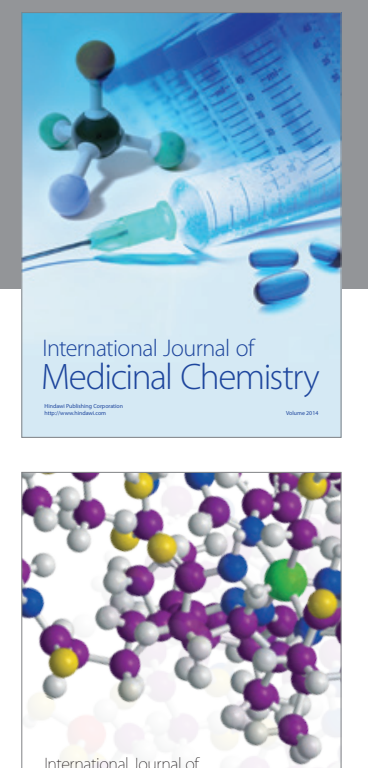

\section{Carbohydrate} Chemistry

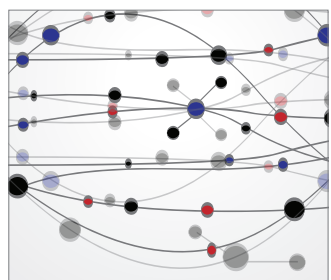

The Scientific World Journal
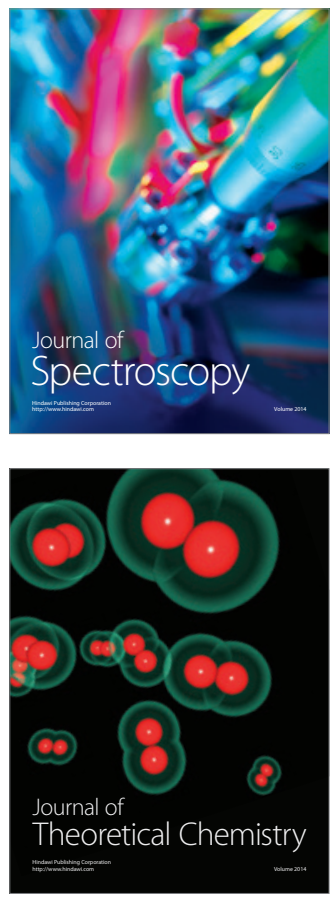
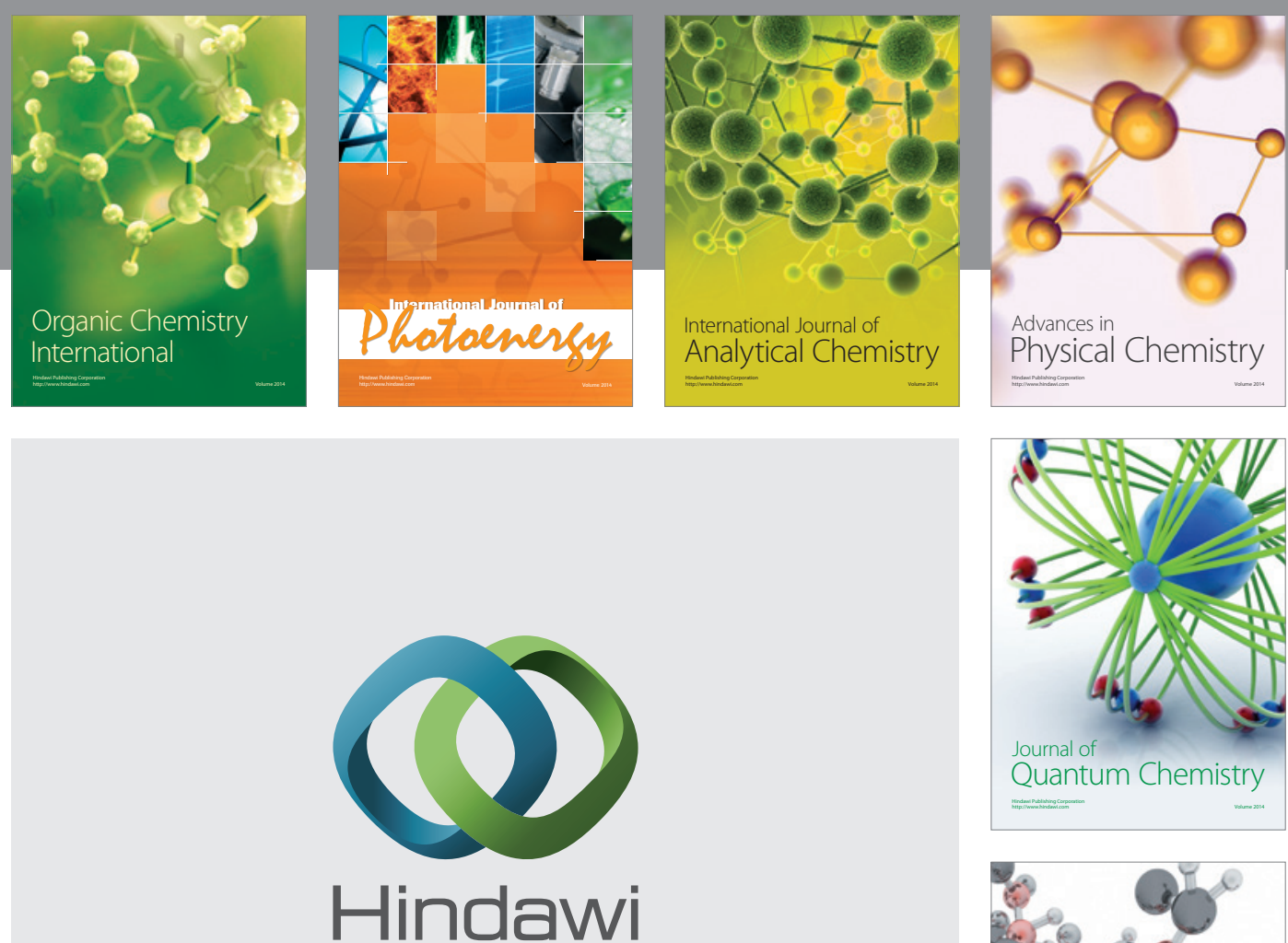

Submit your manuscripts at

http://www.hindawi.com

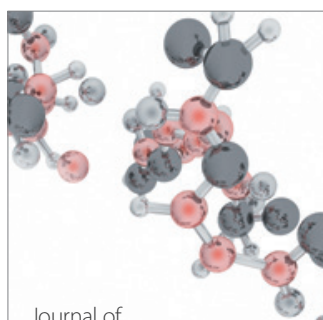

Analytical Methods

in Chemistry

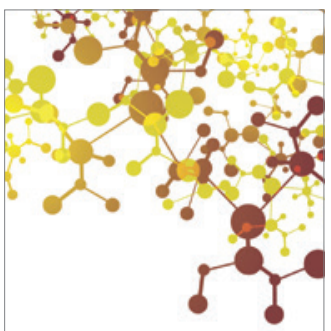

Journal of

Applied Chemistry

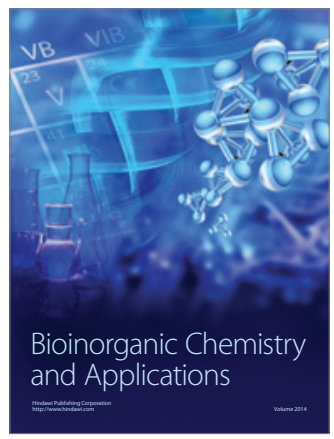

Inorganic Chemistry
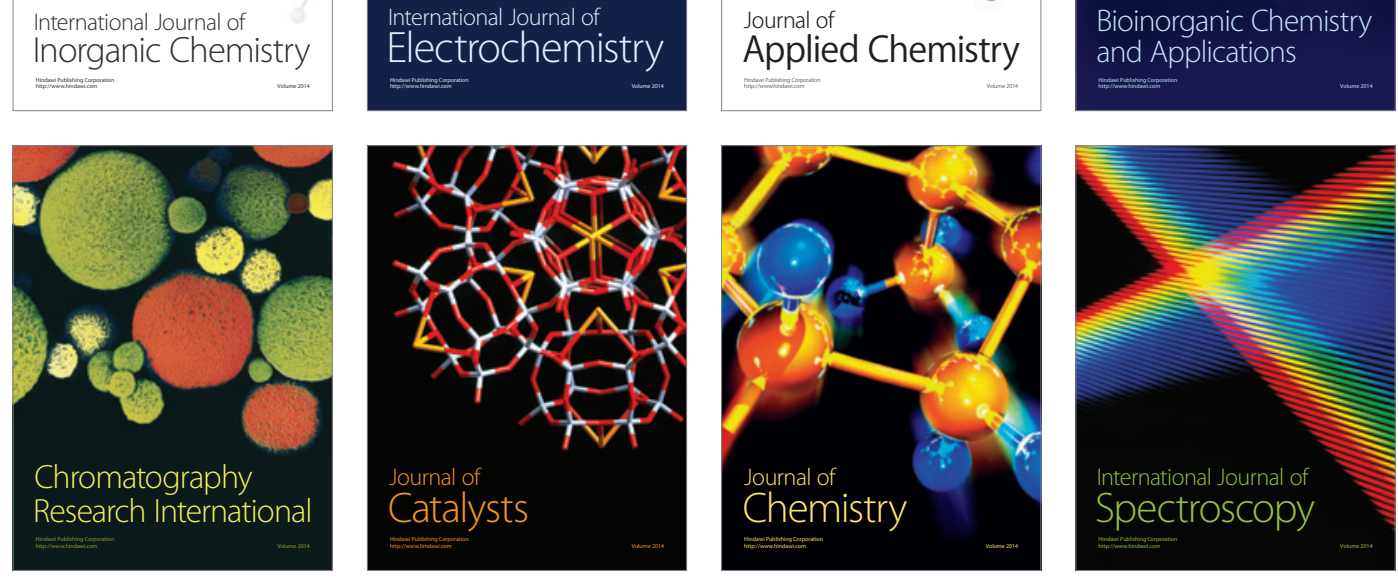